\title{
Investigating how students relate inner products and quantum probabilities
}

\author{
Tong Wan, ${ }^{1}$ Paul J. Emigh, ${ }^{2}$ and Peter S. Shaffer ${ }^{3}$ \\ ${ }^{1}$ Department of Physics, University of Central Florida, Orlando, Florida 32816, USA \\ ${ }^{2}$ Department of Physics, Oregon State University, Corvallis, Oregon 97330, USA \\ ${ }^{3}$ Department of Physics, University of Washington, Seattle, Washington 98195, USA
}

(Received 31 October 2018; published 12 March 2019)

\begin{abstract}
The Born rule, which describes the formalism for determining probabilities, is one of the most fundamental postulates in quantum mechanics. This paper presents results from an investigation into how students apply the Born rule to determine probabilities for energy and position measurements. The investigation includes two stages with independent methods: a quantitative analysis of student written work and a qualitative analysis of student individual interviews. The data from written tasks suggest that after instruction many students have not developed a coherent model for determining probabilities that they can apply to observables regardless of whether the eigenvalues are discrete or continuous. Moreover, many students seem to lack a functional understanding of quantum states and inner products that allows them to translate between Dirac notation and wave function representation. These results motivate student interviews, which allow us to probe student reasoning in depth. Prior research suggests that various features of each notation used in quantum mechanics may have an impact on how students perform computations. We postulate that the features of quantum notations may also interact with student sensemaking. Therefore, we analyze student interviews through the lens of the structural features of quantum notations framework. In particular, we discuss how different structural features may facilitate or hinder student sensemaking about concepts relevant to determining probabilities. The results from both quantitative and qualitative data suggest that unsuccessfully differentiating between a wave function and its associated state vector in Dirac notation may be a primary barrier for students to develop a model for determining probabilities for discrete and continuous cases.
\end{abstract}

DOI: 10.1103/PhysRevPhysEducRes.15.010117

\section{INTRODUCTION}

The Born rule describes how the probabilities for any observable can be determined in a unified way whether the eigenvalues are discrete, continuous, or a mixture of both. It is introduced at the beginning of many textbooks, typically using either a position or a spin context. For example, Griffiths [1] discusses Born's statistical interpretation of the position-space wave function in Chap. 1 and the generalized statistical interpretation in Chap. 3. Both Townsend [2] and McIntyre [3] start by presenting results from SternGerlach experiments that motivate several postulates including one that describes how probabilities are determined for observables with discrete eigenvalues. When the position-space wave function is introduced, they both draw an analogy from the probability amplitudes for observables with discrete eigenvalues. Although these textbooks use

Published by the American Physical Society under the terms of the Creative Commons Attribution 4.0 International license. Further distribution of this work must maintain attribution to the author(s) and the published article's title, journal citation, and DOI. different approaches (position first versus spins first), they all emphasize Born's generalized statistical interpretation.

There is now a substantial body of research on student understanding of quantum mechanics [4-21]. A common finding is that many students struggle to grasp some fundamental ideas throughout their progression from sophomore- to junior- and even to graduate-level quantum mechanics. The results suggest that many students lack a basic model that incorporates some of the key fundamentals of quantum mechanics.

In order for students to construct a coherent model for determining probabilities, they must develop a functional understanding of quantum states and inner products. Students typically encounter both Dirac notation and wave functions, and are expected to be able to solve problems with both. Marshman and Singh [22-24] identified various difficulties that students have with Dirac notation and with translating quantum states from Dirac notation to wave function representation, some of which appear to impact student ability to apply the Born rule.

Differences in how each notation (or algebraic representation) conveys the underlying information can also have an impact on how students perform computations. Gire and Price categorized certain structural features of 
common algebraic quantum notations: Dirac notation, algebraic wave function notation, and matrix notation [25]. They noted that Dirac notation, for example, is often very compact and can be regarded as providing a high degree of symbolic support for computation; wave function notation, on the other hand, provides little explicit support for computation but contains detailed information about the mathematical behavior of a system.

Although prior research has documented student difficulty with determining probabilities in quantum mechanics, we are not aware of any studies that specifically focus on the extent to which students recognize that probabilities for observables with discrete and/or continuous eigenvalues can be determined using analogous procedures. The study presented in this paper is part of our efforts into improving student sensemaking and model building of quantum states and inner products [7-9,11,19,21]. In particular, this study is intended to answer two questions:

(1) What may be the underlying barrier(s) for students to build a coherent model for determining probabilities regardless of whether the observables have discrete or continuous eigenvalues?

(2) What aspects of wave function and Dirac notation can be regarded as supporting or hindering student sensemaking about quantum states and inner products?

Our investigation involves two stages with independent methods. In stage one, we analyze students' written responses to tasks that probe their ability to determine probabilities. We observe the extent to which students recognize that the procedures for determining probabilities for discrete and continuous cases are analogous. We also discuss specific aspects related to quantum states and inner products with which students struggle. The results suggest that reasoning may be influenced by many aspects, such as students' conceptual understanding, grasp of mathematical formalism, and fluency with notations. These aspects seem to be intertwined so that it is difficult to tease out each individual aspect. Therefore, we follow up with a second stage of investigation using individual interviews with students. We analyze interview data through the lens of the structural features of quantum notations framework initially described in Ref. [25]. Although the framework was initially developed in the context of students' performing computations, we postulated that the structural features of those notations may also impact student sensemaking about abstract concepts, such as quantum states and inner products. The qualitative analysis of this study demonstrates how the theoretical framework can be applied to a different context: student sensemaking. Using both quantitative and qualitative methods allows us not only to find the prevalence of common student lines of reasoning but also to understand in depth how the notations interact with student reasoning. Lastly, we triangulate the findings from the quantitative and qualitative analyses.

This paper has six major sections. In Sec. II, we briefly review the background content knowledge required to apply the Born rule. Section III discussed the methods used in this study. Section IV presents results from studentwritten work on tasks used to probe student ability to apply the Born rule and to make sense of inner products. Section V contains an analysis of student interviews using the structural features framework. We conclude with a discussion on the findings convergent from both the quantitative and qualitative data, as well as the implications for instruction in Sec. VI.

\section{BACKGROUND}

This section describes the mathematical background that underlies the application of the Born rule. The discussion is based on the infinite square-well potential since that was the context of the tasks used to probe student reasoning.

The Born rule involves several ideas related to the fundamental postulates of quantum mechanics. McIntyre's textbook [3], for example, lists these postulates as follows: (i) a physical observable is represented mathematically by an operator $\hat{A}$ that acts on kets, (ii) the only possible result of a measurement of an observable is one of the eigenvalues $a_{n}$ of the corresponding operator $\hat{A}$, and (iii) the probability of obtaining the eigenvalue $a_{n}$ in a measurement of the observable $\hat{A}$ (with discrete eigenvalues) on the system in the state $|\psi\rangle$ is $\left|\left\langle a_{n} \mid \psi\right\rangle\right|^{2}$.

Discrete case: The energy eigenvalues for the infinite square-well system are discrete. The energy eigenstates, denoted by $\left|\varphi_{n}\right\rangle$, form a complete basis for this system. Thus, any state vector $|\psi\rangle$ can be expressed as a linear combination of energy eigenstates: $|\psi\rangle=\sum_{n} c_{n}\left|\varphi_{n}\right\rangle$. The coefficient associated with the $n$th energy eigenstate, $c_{n}$, can be determined by taking the inner product between that energy eigenstate and the state vector $\left\langle\varphi_{n} \mid \psi\right\rangle$. By the Born rule, the modulus squared of the inner product $\left|c_{n}\right|^{2}=$ $\left|\left\langle\varphi_{n} \mid \psi\right\rangle\right|^{2}$ gives the probability of measuring that energy.

A wave function can also be used to describe the state of a quantum system, and some wave functions may not be written as an explicit linear combination of energy eigenfunctions. In this case, in order to compute the coefficients, one can convert from Dirac to wave function notation. For the infinite square-well potential that extends from $x=0$ to $x=a$, this becomes $c_{n}=\left\langle\varphi_{n} \mid \psi\right\rangle=\int_{0}^{a} \varphi_{n}^{*}(x) \psi(x) d x$.

Continuous case: The position eigenvalues are continuous and the associated eigenstates form a complete basis. Thus, any state vector can be expanded in terms of the position eigenstates: $|\psi\rangle=\int_{0}^{a} c(x)|x\rangle d x$. In this case, an integral is needed (instead of a sum) because the position eigenvalues are continuous. Since the wave function is defined as the probability amplitude associated with a position $x$, the coefficient $c(x)$ is the position-space wave function, which is typically written as $\psi(x)$. The wave function can also be expressed as the inner product between a position eigenstate and the state vector $\langle x \mid \psi\rangle$. The probability of finding the particle in an infinitesimal region 
$d x$ is $|\langle x \mid \psi\rangle|^{2} d x=|\psi(x)|^{2} d x$. Thus, the probability of finding the particle in a specified region between $x=x_{1}$ and $x=x_{2}$ is an integral $\int_{x_{1}}^{x_{2}}|\psi(x)|^{2} d x$.

As illustrated above, although the energy for a particle in the infinite square-well potential is discrete and the position is continuous, the methods for determining the corresponding probabilities are analogous. Each involves a modulus squared of an inner product. To find the probability of measuring energy (position), one takes the inner product between an energy (position) eigenstate and the state vector. Since the position is continuous, one must integrate over a specified region to find a noninfinitesimal probability.

Before the invention of Dirac notation, state vectors and wave functions were used separately: state vectors for Heisenberg's matrix mechanics and wave functions for Schrödinger's wave mechanics. Dirac notation is a way of unifying ideas from matrix and wave mechanics [26]. Textbooks and physics faculty expect that students recognize quantum mechanics as a coherent theory and are able to determine probabilities for measuring any common observable [27,28]. Thus, it is important that students recognize the similarities between the discrete and the continuous case. In both cases, taking an inner product between the eigenstate of an observable and the state vector is a key step.

\section{METHODS}

This study makes use of two independent methods: (i) a quantitative analysis of student responses to written tasks and (ii) a qualitative analysis of interview transcripts. For the quantitative method, written tasks were designed and administered on exams and online surveys. Student written responses were then analyzed and common lines of reasoning were identified. For the qualitative method, individual student interviews were conducted and transcribed. We analyze the interview transcripts through the lens of the structural features of quantum notations framework initially described in Ref. [25]. In particular, we probe how the structural features of the notations interact with student sensemaking. Below we give an overview of the theoretical framework.

\section{A. Theoretical framework}

Different representations are commonly used in physics. Quantum mechanics, in particular, involves functions, graphs, matrices, and Dirac notation. There is evidence from prior research that student performance can be influenced by the representations that are used [25,29]. Heckler and Scaife, for example, found that student performance on adding and subtracting vectors in (algebraic) $i j k$ format is significantly better than that in (graphical) arrow format.

Gire and Price [25] probed the extent to which different notations (Dirac notation, algebraic wave function notation, and matrix notation) impact student computation in quantum mechanics. They conducted interviews with students who were asked to use multiple notations to represent a superposition state and then to compute the expectation value of the energy. Their observations and analysis suggested four characteristics of the notations that might play a role in student performance. They called these individuation, externalization, compactness, and symbolic support for computation.

There may be different opinions on whether wave function should be considered as a notational system since a position-space wave function, for example, differs from a state vector (which is often represented by a ket in Dirac notation or a column vector in matrix notation). Because a position-space wave function is equal to the inner product between the position eigenstate and a state vector, a position-space wave function is a collection of the coefficients of a state vector in the position space. We argue that although a wave function does not represent a state vector in exactly the same way that a ket or a column vector does, Dirac and matrix notations, as well as wave function are three common ways to convey information about quantum states algebraically. Upper-level physics students are expected to be able to use all three notations. Therefore, examining the structural features of these notations can provide insights into student reasoning.

Below, we discuss the four characteristics of the notations identified by Gire and Price [25]. We do not discuss matrix notation because it is not commonly used for the infinite square-well potential (the context we used to probe student understanding) and no students used this notation on the tasks we gave.

Individuation is "the degree to which important features are represented as separate and elemental (i.e., as an indivisible item)." [25] Gire and Price consider Dirac notation to be highly individuated and wave function notation to be less individuated. In Dirac notation, a quantum state written in terms of basis states (e.g., $\left.a\left|\varphi_{1}\right\rangle+b\left|\varphi_{2}\right\rangle\right)$ can be thought of as having a high degree of individuation since the basis states are represented by individual kets and the probability amplitudes can be easily identified. In contrast, in wave function notation, a linear combination of eigenfunctions is usually compressed into an expression where the basis states are not visible. For example, a superposition of energy eigenstates for a particle in the infinite square well may be expressed as a polynomial, e.g., $\psi(x)=\sqrt{30 / a^{5}}\left(x^{2}-a x\right)$. In this case, the wave function notation is less individuated for the energy eigenstates. Since the probability amplitudes for the energy eigenstates are invisible, this notation provides little help for students to determine the probabilities for energy. However, we argue that wave function notation can be regarded as (relatively) highly individuated for the position basis since a wave function is a collection of the coefficients in the position basis. Although the position basis states are invisible, the corresponding coefficients can be 
easily identified. Therefore, we consider (position-space) wave function notation highly individuated for the position eigenstates but low individuated for other eigenstates.

Externalization "describes the degree to which elements and features are externalized with markings included in the representation." [25] Wave function notation can be regarded as highly externalized, since it makes explicit the detailed mathematical behavior of the quantum states. For example, the wave function $\sqrt{30 / a^{5}}\left(x^{2}-a x\right)$ is a polynomial and the energy eigenfunctions for the infinite square well, $\sqrt{2 / a} \sin (n \pi x / a)$, are sinusoidal. With these functions, one can directly perform a variety of mathematical operations. In contrast, the externalization of Dirac notation is low. Quantum states are represented by kets and the mathematical behavior is implicit. A superposition of energy eigenstates in Dirac notation, $|\psi\rangle=a\left|\varphi_{1}\right\rangle+b\left|\varphi_{2}\right\rangle$, does not explicitly show the mathematical behavior of the state.

Compactness is "a measure of how much space and writing is needed." [25] The relative compactness of two notations may vary. For example, in Dirac notation, a generic state vector $|\psi\rangle$ or a basis state represented by a single ket (e.g., an energy eigenstate is $\left|\varphi_{n}\right\rangle$ ), has higher compactness than the same state in wave function notation [e.g., an energy eigenfunction for the infinite square well is $\sqrt{2 / a} \sin (n \pi x / a)]$. In contrast, a superposition state in wave function notation may have high compactness, if the expression is algebraically simplified. For example, $\psi(x)=$ $\sqrt{30 / a^{5}}\left(x^{2}-a x\right)$, a polynomial with only two terms, contains an infinite number of superimposed energy eigenstates. Moreover, a superposition state with many nonzero expansion coefficients in Dirac notation may have low compactness if the coefficients are expressed numerically or high compactness if the coefficients are specified generically (i.e., as $\sum_{n} c_{n}\left|\varphi_{n}\right\rangle$ ).

Symbolic support for computation measures the degree to which the physical shape of a notation system supports computation. Dirac notation is considered to have a high degree of symbolic support. The bras and kets have asymmetric shapes: a straight vertical line on one side and an angle bracket on the other side: |\rangle or \langle| . In this notation, it is clear (to those who have experience with Dirac notation) that any expression with a single unclosed bracket represents a vector in some vector space (e.g., Hilbert space). The asymmetry of unclosed brackets provides visual cues for the different sequences of elements in different operations: inner products and outer products. An inner product is denoted by a closed bracket, $\langle\mid\rangle$, resulting in a scalar, whereas an outer product has a different sequence of elements, |\rangle\langle| , resulting in an operator. In contrast, the wave function notation provides little symbolic support for computation. Gire and Price gave an example in which a student immediately wrote down in Dirac notation $\langle\psi|\hat{H}| \psi\rangle$ when asked to calculate the expectation value of the energy. When the interviewer prompted the student to use wave function notation, the student admitted that he did not remember how to compute in that notation. After the interviewer hinted that there were integrals involved, the student incorrectly wrote $\int \psi \psi^{*} \hat{H} d x$. Overall, their interviewees tended to make more errors with the order of elements when they were using wave function notation.

\section{B. Context for research}

The research described in this paper has been primarily carried out in the junior-level quantum mechanics course at the University of Washington (UW). The course is a two-quarter sequence. The first quarter covers Chap. 1-4 of Griffiths' Introduction to Quantum Mechanics [1]. The rest of the book is finished in the second quarter. The typical enrollment ranges from 70 to 110 students during this study.

The course meets weekly for two 80-min lectures and a 50-min small-group (15-30 students) section. The smallgroup section uses Tutorials in Physics: Quantum Mechanics [30] developed by the Physics Education Group at the UW. It is comprised of three components: (i) an online-survey (also known as the tutorial pretest), (ii) an in-class worksheet, and (iii) homework. Before students attend the small-group section, they are asked to complete an online survey with a series of questions. Students receive credit for participation, but the responses are not graded for correctness.

Most of the junior-level students (except for transfer students) have had completed the sophomore-level quantum mechanics course at the UW. The sophomore-level course covers the first five (or six) chapters of McIntyre's textbook Quantum Mechanics: A Paradigms Approach [2]. Therefore, most of the students in this study have experienced both position-first (Griffiths'textbook [1]) and spins-first (McIntyre's textbook [3]) approaches. The sophomore-level course implements the tutorials [30] in the lecture setting, facilitated by the lecture instructor and several graduate teaching assistants.

\section{STUDENT ABILITY TO DETERMINE PROBABILITIES}

In this section, we first present the written tasks and give an overview of student performance. We then discuss in detail the productive strategies students use for determining probabilities as well as specific aspects related to quantum states and inner products with which students struggle.

\section{A. Written tasks}

Three of the research tasks used to probe student ability to find probabilities are shown in Fig. 1. These tasks are related to energy and position measurements. Although we have also used tasks on momentum measurement, the results are similar to those on position measurement. Therefore, we 
Task 1

Consider a particle in a quantum mechanical infinite square well of width $a$. The normalized wave function for the particle is given by $\Psi(x, t=0)=\sqrt{\frac{30}{a^{5}}}\left(x^{2}-a x\right)$ for $0<x<a$ at $t=0$.

Do not evaluate any integrals.

A. Suppose you were to measure the energy of this particle at $t=0$. Determine the probability that the energy is equal to $E_{1}$, the energy of the ground state. Explain.

B. Suppose you were to measure the position of this particle at $t=0$. Determine the probability that the position of the particle is measured to be between $x=0$ and $x=a / 3$. Explain.
Task 2

Consider a particle in an infinite square well that extends from 0 to $a$. The state of the particle at $t=0$ can be described as $|\psi\rangle=\frac{1}{\sqrt{2}}\left|\varphi_{2}\right\rangle+\frac{1}{\sqrt{2}}\left|\varphi_{3}\right\rangle$, where $\left|\varphi_{n}\right\rangle$ represents the $n$th energy eigenstate.

Which of the expression(s) below can represent the probability of finding the particle in the left half of the well at $t=0$. Check all that apply. (Assume the wave functions below are all real.)
A. $\int_{0}^{a / 2}\langle\psi \mid \psi\rangle d x$
B. $\int_{0}^{a / 2} \psi^{2}(x) d x$
C. $\int_{0}^{a / 2}\left(\frac{1}{2} \varphi_{2}^{2}(x)+\frac{1}{2} \varphi_{3}^{2}(x)\right) d x$
D. $\int_{0}^{a / 2}\left(\frac{1}{2} \varphi_{2}^{2}(x)+\frac{1}{2} \varphi_{3}^{2}(x)+\varphi_{2}(x) \varphi_{3}(x)\right) d x$

Task 3

Indicate all of the following equations that are correct.

(a) $\langle x \mid \psi\rangle=\int x \psi(x) d x$

(b) $\langle x \mid \psi\rangle=\int \delta\left(x-x^{\prime}\right) \psi\left(x^{\prime}\right) d x^{\prime}$

FIG. 1. Tasks on determining probabilities and translating inner products from Dirac notation to wave function notation.

only present tasks that focus on energy and position measurements in this paper.

Task 1 was part of a midterm exam in the first quarter of the junior-level course taken by 71 students [31]. The exam occurred after lecture instruction on the first three chapters in Griffiths [1] as well as after tutorial instruction that primarily targeted the ideas of time dependence and the effects of quantum measurements [9].

Both task 2 and task 3 were in multiple-choice format. They were given as part of the online surveys in the juniorlevel course after lecture instruction on the first three chapters of Griffiths [1] as well as after a preliminary version of a set of tutorials [21] intended to help students develop a model for determine probabilities.

Before the multiple-choice version of task 2 was designed, a free-response version (in which the probability density instead of the probability in a specified region was asked) was given on a final exam in the sophomore-level course. Student written responses were analyzed and the common incorrect answers were identified. Based on the common incorrect answers generated by the students, we designed the answer options to the multiple-choice version. There were 76 students ( $70 \%$ of the students enrolled) answered task 2 and 57 students (53\% of the students enrolled) agreed to participate in this study.

Task 3 was adapted from a question used by Singh and Marshman [22,23]. It was given in three academic quarters. There were 161 students (75\% of the students enrolled) answered and 121 students (57\% of the students enrolled) agreed to participate in this study.

\section{Task 1}

On task 1, students are given the wave function of a particle in an infinite square well. The wave function is not explicitly written as a superposition of energy eigenfunctions. Students are asked to determine the probability of measuring the ground state energy and the probability of finding the particle in a specified region. They are told that it is not necessary to evaluate any integrals.

According to the Born rule, the probability of measuring the ground state energy is the modulus squared of the inner product between the ground state and the state vector, $\left|\left\langle\varphi_{1} \mid \psi\right\rangle\right|^{2}$. To determine the probability, one needs to convert the inner product from Dirac notation to wave function notation:

$$
\left|\left\langle\varphi_{1} \mid \psi\right\rangle\right|^{2}=\left|\int_{0}^{a} \varphi_{1}^{*}(x) \psi(x) d x\right|^{2} .
$$

To find the position probability, one can integrate the probability density over the specified region:

$$
\int_{0}^{a / 3}|\langle x \mid \psi\rangle|^{2} d x=\int_{0}^{a / 3}|\psi(x)|^{2} d x .
$$

Since the state of the particle is given in wave function notation, students only needed to write down the righthand side of the equation above for their answer to be regarded as correct.

\section{Task 2}

On task 2, students consider a quantum state written in terms of the energy eigenstates for an infinite square well. They are asked to choose the expression(s) that can represent the probability of finding the particle in the left half of the well. Since the quantum state is written in Dirac notation, one needs to convert the quantum state from Dirac notation to wave function notation: 


$$
\psi(x)=\frac{1}{\sqrt{2}}\left\langle x \mid \varphi_{2}\right\rangle+\frac{1}{\sqrt{2}}\left\langle x \mid \varphi_{3}\right\rangle=\frac{1}{\sqrt{2}} \varphi_{2}(x)+\frac{1}{\sqrt{2}} \varphi_{3}(x) .
$$

By convention, the energy eigenfunctions for a particle in the infinite square well are usually taken to be real functions. Thus, the probability density is

$$
|\psi(x)|^{2}=\frac{1}{2} \varphi_{2}^{2}(x)+\frac{1}{2} \varphi_{3}^{2}(x)+\varphi_{2}(x) \varphi_{3}(x) .
$$

The probability of finding the particle in the left half of the well is therefore

$$
\int_{0}^{a / 2}\left[\frac{1}{2} \varphi_{2}^{2}(x)+\frac{1}{2} \varphi_{3}^{2}(x)+\varphi_{2}(x) \varphi_{3}(x)\right] d x
$$

Both answers B and D are correct.

\section{Task 3}

On task 3, students are asked to indicate the equation(s) that correctly translate the inner product from Dirac to wave function notation. Although this task does not directly ask for probability, it probes whether students recognize that the wave function is an inner product and whether students are able to translate the inner product from Dirac to wave function notation. To answer this question, students need to identify the corresponding position-space wave functions for states $|x\rangle$ and $|\psi\rangle$. For a position eigenstate $\left|x^{\prime}\right\rangle$, the position-space wave function is a one-dimensional delta function since $\left\langle x \mid x^{\prime}\right\rangle=\delta\left(x-x^{\prime}\right)$. The result of the inner product between two state vectors is equal to the inner product between the corresponding two wave functions. Alternatively, students can use the definition of the wave function in Dirac notation, $\psi(x)=\langle x \mid \psi\rangle$ and check whether the integral results in $\psi(x)$. Answer (b) is therefore correct.

\section{B. Overall performance}

Table I shows the percentage of correct responses to each task. Thirty-seven students $(52 \%, N=71)$ gave correct expressions for the probability of measuring the ground state energy (task 1A). Eleven students $(15 \%, N=71)$ correctly stated that $\left|c_{1}\right|^{2}$ gives the probability (where $c_{1}$ is the first expansion coefficient in the energy basis). Some of them determined the coefficient using incorrect methods (e.g., acting the Hamiltonian on the wave function), and

TABLE I. Percentage of correct answer to each question on the written tasks.

\begin{tabular}{lcc}
\hline \hline Task & Number of participants & Correct answer \\
\hline 1A & $N=71$ & $52 \%$ \\
1B & $N=71$ & $58 \%$ \\
2 & $N=57$ & $32 \%$ \\
3 & $N=121$ & $49 \%$ \\
\hline \hline
\end{tabular}

others did not describe how to determine $c_{1}$. Since the wave function is not explicitly written in terms of the energy eigenfunctions, we considered a response to be completely correct only if it explained how the coefficient can be determined using an inner product in either Dirac or wave function notation. Common incorrect answers included expressions for the expectation value of the energy and the modulus squared of the normalization constant.

On task 1B, forty-one students $(58 \%, N=71)$ gave correct expressions for the probability of finding the particle in the specified region. The most common incorrect answer was the expectation value for position given by 10 students $(14 \%, N=71)$. Another 10 students $(14 \%$, $N=71$ ) wrote integrals with different incorrect integrands, such as $\int_{0}^{a / 3} x \psi(x) d x$. Only 24 students $(34 \%, N=71)$ gave correct answers to both parts of task 1 .

On task 2 , only 18 students $(32 \%, N=57)$ gave correct answers for the position probability. We define a correct answer as one in which students chose at least one correct expression but did not choose any incorrect expressions. Many students seemed to know the definition of probability density since they chose the expression that has $|\psi(x)|^{2}$ (answer B) as the integrand. However, thirty-three students $(58 \%, N=57)$ included the incorrect expression $\int_{0}^{a / 2}\langle\psi \mid \psi\rangle d x$ (answer A) in their answers. The percentage of students who included each of the answers is shown in Table II.

The percentage of correct answer on task 3 are aggregated across different academic quarters since the difference for any pair of data sets is not statistically significant ( $p>0.05$, Fisher's exact test). Only 59 students (49\%, $N=121$ ) were able to identify answer (b) as the only correct equation for the inner product. There were 52 students $(43 \%, N=121)$ included answer (a) in their

TABLE II. Percentage of each answer chose by students on task 2. Students were allowed to choose more than one answer.

\begin{tabular}{ll}
\hline \hline $\begin{array}{l}\text { Answer } \\
\text { choice }\end{array}$ & $\begin{array}{c}\text { Percentage } \\
(N=57)\end{array}$ \\
\hline A & $58 \%$ \\
B & $67 \%$ \\
C & $26 \%$ \\
D & $63 \%$ \\
\hline \hline
\end{tabular}

TABLE III. Percentage of each answer chose by students on task 3. Students were allowed to choose more than one answer.

\begin{tabular}{lc}
\hline \hline $\begin{array}{l}\text { Answer } \\
\text { choice }\end{array}$ & $\begin{array}{c}\text { Percentage } \\
(N=121)\end{array}$ \\
\hline (a) & $43 \%$ \\
(b) & $68 \%$ \\
Neither (a) or (b) & $8 \%$ \\
\hline
\end{tabular}


responses, as shown in Table III. The results are consistent with those obtained by Singh and Marshman [22,23] on a similar question.

\section{Student model for determining probabilities}

To figure out the extent to which students have developed a model for determining probabilities, we examine the responses from students who gave correct expressions for either energy or position probability on task 1 . In particular, we discuss the extent to which students are able to relate inner product and probabilities, and how they make use of Dirac and wave function notations. This allows us to identify the productive strategies used by students who were successful in determining the expressions.

When asked to determine the probability of measuring the ground state energy on task $1 \mathrm{~A}$, about half of the students who gave a correct expression (20 out of 37) started with an expression in Dirac notation and then translated from Dirac to wave function notation. About one-quarter of the students who answered correctly (8 out of 37) used Dirac notation only, with the other quarter (9 out of 37) using wave function notation only. Although the state of the particle is represented by a wave function, students who gave a correct expression for the energy probability were more likely to use Dirac notation.

Figure 2 shows an example student response to task 1A [32]. The student wrote an inner product in Dirac notation first and then used it as a template to rewrite the inner product in wave function notation. This student drew an arrow pointing to the expression in Dirac notation and commented, "use inner product to determine degree to which $\Psi$ overlaps with 1 st eigenstate of square well." The student was using the inner product in Dirac notation to explain their reasoning even if the quantum state in the task is not written in Dirac notation. It seems that Dirac notation helped this student to articulate what the mathematical operation means and how that is related to the physics scenario.

On task 1B, most of the students used wave function notation only. This result is not necessarily surprising, as the state is already written in wave function notation. To determine the probability of finding the particle in a specified region, one can simply integrate the modulus squared of the wave function over the specified region. However, eight students $(11 \%, N=71)$ explicitly wrote the analogous inner product $\langle x \mid \psi\rangle$ and used it as a template in the same way. Most of these students (6 out of 8 )

$$
\begin{aligned}
& P\left(E_{1}\right)=\mid\left\langle\psi_{1} \mid \Psi(x, 0)\right\rangle^{2} \longleftarrow \begin{array}{l}
\text { use inner product to } \\
\text { determine degree to which }
\end{array} \\
& =\left|\int_{-\infty}^{\infty} \sqrt{\frac{2}{a}} \sin \frac{\pi x}{a} \sqrt{\frac{15}{a^{5}}}\left(x^{2}-a x\right) d x\right|^{2} \text { of square wareal. }
\end{aligned}
$$

FIG. 2. An example of correct responses to task 1A. correctly translated $\langle x \mid \psi\rangle$ into wave function notation. One of them answered:

$$
\begin{aligned}
& \text { "By generalized statistical interpretation again, }\langle x \mid \psi\rangle= \\
& \psi(x)=c(x) . \text { Because } c(x) \text { is continuous, }|c(x)|^{2} d x \\
& \text { constitutes the probability over some } d x \text {. So, by integra- } \\
& \text { tion, } P=\int_{0}^{a / 3}|c(x)|^{2} d x=\int_{0}^{a / 3}|\psi(x)|^{2} d x . "
\end{aligned}
$$

This student correctly recognized that the inner product is the wave function, which is also the coefficient in position space. The six students who correctly translated $\langle x \mid \psi\rangle$ into wave function notation also correctly used inner products as templates for the energy probability. These students appear to have a coherent model for determining probabilities. They recognized that determining probabilities for both discrete and continuous cases involve inner products.

\section{Specific difficulties students have with quantum states and inner products}

Our analysis of the written responses also revealed some incorrect patterns in how students reason about inner products when finding probabilities. We use the term "difficulty" to refer to these incorrect lines of reasoning. In this section, we identify three such difficulties and discuss example responses to demonstrate student reasoning for each difficulty in detail.

\section{Failure to recognize that the expansion coefficients can be determined using inner products}

On task $1 \mathrm{~A}$, eleven students $(15 \%, N=71)$ answered that the probability of measuring the ground state energy is $\left|c_{1}\right|^{2}$. Not every student explained what $c_{1}$ refers to and those who did stated that $c_{1}$ is the first expansion coefficient in the energy basis. Seven out of eleven students determined $c_{1}$ with incorrect methods. Some of them acted the Hamiltonian on the wave function, and some claimed that the expansion coefficient equals the normalization constant. These students do not recognize that the expansion coefficients are supposed to be determined by taking the inner products between the energy eigenstates and the state vector.

Four out of eleven students did not attempt to determine the expansion coefficient. For example, one of the students answered that "Probability of finding particle with $E_{1}$ energy depends on how much $\psi_{1}$ contributes to $\Psi$, which can be found by squaring coefficient of $\psi_{1}$." We speculated these students may not realize how the expansion coefficient can be determined.

Similar to the responses without an attempt to determine the expansion coefficient, one student explicitly stated that the probability cannot be determined:

"The probability of finding the particle in its ground state energy would depend on how the wave function is composed of its energy eigenstates, so we cannot tell." 
This student correctly recognized that the probability depends on "how the wave function is composed of its energy eigenstates." However, the student incorrectly concluded that they could not determine the probability. It is likely because the given wave function is not explicitly written as a linear combination of energy eigenfunctions. This is consistent with what we have found previously that many students incorrectly assumed that the state represented in a particular basis does not provide information about observables corresponding to other bases $[19,21]$.

It has been our experience that students usually are able to determine probabilities when the state is expressed in the basis corresponding to the observable of interest. However, many fail to recognize that an inner product can be used when the state is not written in the basis of interest.

\section{Tendency to confuse quantum state vectors with wave functions}

On task 2, thirty-eight students $(67 \%, N=57)$ correctly identified that the probability is given by $\int_{0}^{a / 2}|\psi(x)|^{2} d x$. However, more than half of all students $(58 \%, N=57)$ chose the expression $\int_{0}^{a / 2}\langle\psi \mid \psi\rangle d x$. Many of these students stated that $\langle\psi \mid \psi\rangle$ and $|\psi(x)|^{2}$ are equivalent. A student response is quoted below:

"Bra(Psi)ket(Psi) $[\langle\psi \mid \psi\rangle]$ is the equivalent of the complex conjugate of Psi times Psi [ $\left.\psi^{*} \psi\right]$ and is the definition of the integrand for probability. When expanded, it becomes [answer] D."

This student appears to be making a comparison between the two expressions based on their structures. The inner product $\langle\psi \mid \psi\rangle$ can be considered as the product of the bra $\langle\psi|$ (the dual state of $|\psi\rangle$ ) and the ket $|\psi\rangle$. Similarly, the modulus squared of the wave function is the product of $\psi^{*}(x)$ (the complex conjugate of wave function) and $\psi(x)$. Both expressions are products of a mathematical object and its conjugate. This student incorrectly concluded that those expressions are equivalent.

We also found that some students explicitly call both integrands in answers A and B the modulus squared of the wave function. For instance, "A and B are the same, the modulus squared of the wave function, giving the probability density, from 0 to $a / 2$." This student does not appear to differentiate between the state vector and its associated wave function.

Fifteen students $(26 \%, N=57)$ included answer choice $\int_{0}^{a / 2}\left[\frac{1}{2} \varphi_{2}^{2}(x)+\frac{1}{2} \varphi_{3}^{2}(x)\right] d x$. Many of these students stated that this expression is equal to $\int_{0}^{a / 2}\left[\frac{1}{2} \varphi_{2}^{2}(x)+\frac{1}{2} \varphi_{3}^{2}(x)+\right.$ $\left.\varphi_{2}(x) \varphi_{3}(x)\right] d x$. Some students gave their explanations in more detail by stating that the third term is equal to zero because energy eigenstates are orthogonal. An example student response is below:
"The probability of finding a particle at the left half is an integral of the square modulus of the wave function from 0 to a/2, which is [answer] B. Both [answers] $C$ and $D$ are the same because the third term in $D$ is zero. After all, the energy eigenstates are orthogonal."

This student incorrectly stated that the third term in answer $\mathrm{D}$ is zero. It is not clear whether the student meant the product $\varphi_{2}(x) \varphi_{3}(x)$ is zero or the integral $\int_{0}^{a / 2} \varphi_{2}(x) \varphi_{3}(x) d x$ is zero. It could be that the student was confusing the product of energy eigenfunctions $\varphi_{2}(x) \varphi_{3}(x)$ with the inner product between two energy eigenstates $\left\langle\varphi_{2} \mid \varphi_{3}\right\rangle$, and therefore arrived at an incorrect conclusion that the product $\varphi_{2}(x) \varphi_{3}(x)$ is zero. It is also likely that the student meant the integral $\int_{0}^{a / 2} \varphi_{2}(x) \varphi_{3}(x) d x$ is zero. The inner product between the two energy eigenstates written in wave function notation is an integral over the entire region: $\int_{0}^{a} \varphi_{2}(x)$ $\varphi_{3}(x) d x$. The integral over the left half of the well is not equal to zero. It seems that this student does not recognize that when using orthogonality between states, an integral may only be calculated when the limits of integration cover the entire region. No matter the student meant the product of the functions is equal to zero or the integral is equal to zero, this student appears to have difficulty with translating the inner product from Dirac to wave function notation.

We noticed that for students who included answer choice $\int_{0}^{a / 2}\left[\frac{1}{2} \varphi_{2}^{2}(x)+\frac{1}{2} \varphi_{3}^{2}(x)\right] d x$, about three-fifths (nine out of fifteen) included answer choice $\int_{0}^{a / 2}\langle\psi \mid \psi\rangle d x$ as well. These students appear to be consistently confusing inner products of state vectors with inner products of functions. They do not differentiate between the inner product $\langle\psi \mid \psi\rangle$ and probability density $\psi^{*}(x) \psi(x)$, and tend to confuse the product of two energy eigenfunctions with the inner product between two energy eigenstates. Some explicitly stated that $\langle\psi \mid \psi\rangle$ is also the modulus squared of wave function, which suggest that they do not differentiate between the state vector and its associated wave function.

\section{Tendency to match symbols when translating inner products from Dirac to wave function notation}

On task 1B, there were some students who incorrectly wrote that $\langle x \mid \psi\rangle=\int x \psi(x) d x$, an error previously noted by Singh and Marshman $[22,23]$. When we asked students to choose the correct integral(s) for $\langle x \mid \psi\rangle$ on task 3, fifty-two students $(43 \%, N=121)$ included the incorrect integral $\int x \psi(x) d x$. Seventeen students $(14 \%, N=121)$ explained that this equation follows the "definition" of an inner product. Some students did not provide detailed explanation, but some elaborated what they meant by the definition of an inner product. An example student response is shown below:

"The inner product of two functions is the integral of their product (with the first component complex 
conjugated) over all values of the differential element just like we see in equation 1 [a]."

This student used the phrase "the inner product of two functions" instead of "the inner product of two state vectors." We speculate that this student is reading off the symbols inside the bracket and interpreting them as functions, rather than considering each unclosed bracket and the symbol(s) together as a single element, which is a state vector. In addition, the student appears to be describing an incorrect template for translating expressions in Dirac notation to wave function notation. In this template, the symbols in the bra-ket ( $x$ and $\psi)$ are matched with the symbols in the integrand $[x \psi(x)]$.

Another relatively common (about $9 \%, N=121$ ) incorrect explanation to task 3 was that the $x$ in the expression $\langle x \mid \psi\rangle$ is the position operator $\hat{x}$ or the eigenvalue of the position operator. Below is an example student response:

"The position operator acts just like regular multiplication, except position is continuous, so we have to integrate when we act the position operator on the state $|\psi\rangle . "$

This student appears to interpret the inner product as a position operator acts on the state $|\psi\rangle$. This inner product is considered as the multiplication between a position $x$ and the state vector. This student seems to confuse the position eigenstate with the position operator.

The results above suggest that when students translate inner products from Dirac to wave function notation, many tend to match the symbols in the bra-ket with those in the integral. Some students do not seem to interpret the bra-ket as the inner product between two quantum states, but rather interpret it as the inner product between two functions denoted by the symbols inside the bra-ket. Others interpret the symbol $x$ as the position operator. These incorrect interpretations suggest that many students tend not to differentiate between expressions denoted by the same symbol (e.g., $\hat{x}, x,\langle x|)$.

\section{E. Summary}

We have analyzed student ability to determine probabilities for discrete and continuous cases as well as to translate between Dirac and wave function notations. The results suggest that only some students appear to recognize that determining probabilities for discrete cases is analogous to determining probabilities for continuous cases. Students who successfully found the expression for the energy probability were more likely to use Dirac notation as a template. The results also suggest that some students did not seem to recognize the expansion coefficient in the energy basis can be determined using an inner product, although they correctly stated that the energy probability depends on the expansion coefficient. When students translated expressions between Dirac and wave function notations, many tended to (i) confuse an inner product of state vectors with a product of wave functions [e.g., confuse $\langle\psi \mid \psi\rangle$ with $\psi^{*}(x) \psi(x)$ and $\left\langle\varphi_{m} \mid \varphi_{n}\right\rangle$ with $\left.\varphi_{m}^{*}(x)_{n}(x)\right]$ and (ii) confuse the position eigenstate vector $|x\rangle$ with the function $x$ or with the position operator $\hat{x}$. These results seem to suggest that many students do not differentiate between state vectors and wave functions. In addition, we have seen students call state vectors (wave) functions on both tasks 2 and 3 .

\section{STUDENT SENSEMAKING ABOUT QUANTUM STATES IN DIFFERENT NOTATIONS}

As discussed in the previous section, the errors students make when reasoning with quantum states and inner products seem to be related to their interpretations of expressions in Dirac and wave function notations. Therefore, we conducted student interviews and attempted to analyze student sensemaking through the lens of the structural features of quantum notations framework [25], which was initially developed to analyze student reasoning when performing computations. In this section, we discuss how the various structural features of Dirac notation and of wave function notation may support and/or hinder student sensemaking about quantum states in Dirac and wave function notations.

\section{A. Interview tasks}

We conducted individual interviews $(N=6)$ near the end of the second quarter of the junior-level quantum course after relevant lecture instruction and also after students had worked through early versions of researchbased tutorials [30] intended to improve student understanding of the concepts discussed in this paper. The interviewees were all the students who volunteered to participate in this study. None of them were the same students who completed the written tasks (discussed in section IV) since the interview tasks were administered in a different academic year. The names of the interviewees included in this paper are pseudonyms.

The interviews were semistructured and used a thinkaloud protocol. Students were asked to talk aloud about their reasoning processes when they were working through the tasks. The interviewer asked open-ended questions to elicit students' ideas and reasoning.

Each interview consisted of two parts. In part 1, students were given the questions in task 1 (See Fig. 1 in Sec. IVA), which asked for energy and position probabilities. After the students had worked through the task, they were asked to compare the approaches they used to determine the probabilities for energy and position measurements. Students were prompted to discuss the similarities and differences between their approaches.

In part 2, students were given expressions related to quantum states and inner products [e.g., $|\psi\rangle, \psi(x)$, and 
$\langle x \mid \psi\rangle]$ one at a time. They were asked to explain what each expression represents. Example prompts include "what does this expression represent" and "what would you call this expression." The interviewer also prompted students to make sense of the expressions by saying: "can you give an interpretation of this expression." For some of the expressions, the interviewer asked students to translate from Dirac to wave function notation and to explain how they decided what the integrand should be. The purpose of these openended questions was to gain more insight into how students make sense of the expressions in tasks 2 and 3.

\section{B. Interview results}

We discuss, for Dirac and wave function notations, how some of the structural features can be regarded as supporting or hindering student reasoning. We present examples that provide insightful details into how some of the structural features may influence student reasoning. This study is not intended to conduct a comprehensive analysis for each of the structural features. The goal is to figure out potential strategies that can be used to address the common errors students make when they reason about quantum states in different notations.

Below we discuss the primary findings of our analysis. We start with the results for wave function notation, followed by the results for Dirac notation.

\section{High individuation of wave function notation for position eigenstates may support students to determine position probabilities}

During the interview, Jack immediately wrote the integral $\int_{0}^{a / 3} \psi^{*}(x) \psi(x) d x$ when asked to determine the position probability. The interviewer then asked him to explain the integral he wrote.

Jack: Well, the boundaries of the integral are, you know, that's saying, you are looking within a region defined by the boundaries of the integral. So zero to a 3 is where we wanna find the probability for. If we set it to a instead of $a / 3$, we will necessarily end up with a probability equals to one and then by, well so it's not actually explaining it but rather it's just explaining my own thought process. This $\left[\psi^{*}(x) \psi(x)\right]$ is the same as the magnitude of $\psi(x)$ squared [he writes $\left.|\psi(x)|^{2}\right]$. And that gives you the probability at position $x$ of that wave function. And so, if you have the probability at position $x$, you integrate from zero to a/3 across $x$, you will end up with the, it's basically the infinitesimal sums of all the tiny little probabilities of every point between zero to $a / 3$.

Jack first explained correctly how he determined the boundaries of the integral. He then stated that $|\psi(x)|^{2}$ gives the probability of finding a particle at position $x$. This statement is not completely correct since $|\psi(x)|^{2}$ gives the probability density and the probability of finding a particle in an infinitesimal region $d x$ around position $x$ would be $|\psi(x)|^{2} d x$. However, Jack was able to relate $|\psi(x)|^{2}$ to the probability associated with the position $x$ and then correctly recognized that the integral is a sum of infinitesimal probabilities.

As discussed in Sec. III, wave function notation is highly individuated for the position eigenstates. The value of a wave function for a particular position $x$ is the coefficient for the corresponding position eigenstate. Therefore, the probability of finding the particle in an interval $d x$ is given by $|\psi(x)|^{2} d x$. The high individuation of wave function notation for the position states appears to support Jack's reasoning with determining position probability. He recognized a need for modulus squared of the coefficient, as well as a sum of the probabilities, which is an integral.

\section{Low symbolic support of wave function notation may hinder student ability to find expansion coefficients}

When Jack was trying to determine the energy probability, he stated that the ground state wave function in task 1 would be the $x$ term in the polynomial.

Jack: But I am used to seeing the $\psi$ functions like trig or something, that's more, when you have the form like [sin] $n \pi x / a$, that makes more sense to me when it's asking about the ground state, so I don't actually know right now or I'm going to commit, I guess the more appropriate answer... But it seems to me that the ground state of this function would be when you have the single, you have the $x^{2}-a x . I$ am thinking the $a$ is the determining factor, or the $x$ is the value that would yield, you know it's like a superposition of states. That's the word I should, I am looking for. The energy $E_{1}$ is what you get when you find the particle in the state defined by the single, you know $x$ instead of $x^{2}$, so the probability would be based off the factor $a$ and then the coefficient in front of the whole thing.

Jack recalled correctly that the energy eigenfunctions for the infinite square well are sine functions. However, he did not recognize the expansion coefficients can be determined by integration. This seems to be consistent with the low symbolic support of wave function notation, which provides little visual cue on what mathematical operation needs to be performed. Moreover, since the given wave function is not expressed in terms of the sine functions, both the energy eigenfunctions and their associated probability amplitudes are invisible. This wave function has a low degree of individuation for the energy eigenstates. The appearance of the polynomial, a combination of two terms, may have cued Jack to state instead that the $x$ term corresponds to the ground state, and the $x^{2}$ term corresponds to the first excited state, as shown in Fig. 3. Then, he incorrectly concluded that the coefficient for the ground 


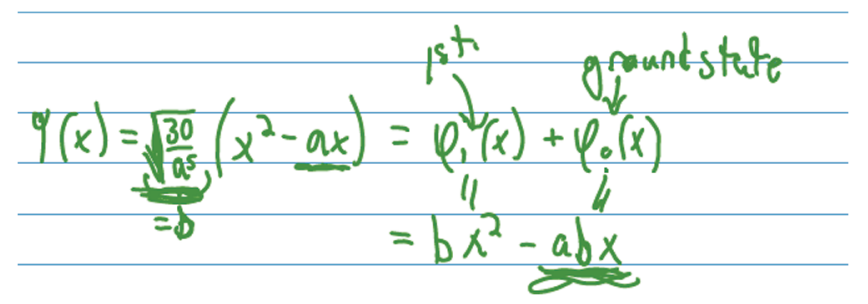

FIG. 3. Jack's written response during the interview.

state is just the coefficient in front of function $x$ and the probability of measuring the ground state energy is the squared of that coefficient. The low individuation of wave function notation for the energy basis seems to have had a negative impact on Jack's reasoning.

The results above seem to suggest that using wave function notation only may not be a productive strategy for determining expansion coefficients for energy eigenstates. This seems consistent with the low individuation for the energy eigenstates and low symbolic support of wave function notation. When the wave function is compressed into a polynomial, the energy eigenfunctions and their associated coefficients are highly encoded. Compared to Dirac notation, wave function notation provides little visual cue on how the coefficients can be determined. Although experts may recognize an integral is needed to determine the coefficient, students do not seem to have developed this expertise.

\section{High symbolic support of Dirac notation may facilitate student sensemaking of inner products}

When Amy was working on Task 1, she correctly stated that the wave function could be written as a linear combination of the energy eigenfunctions. She also referenced the Born rule and argued that the modulus squared of the first coefficient gives the probability for the ground state energy. However, she was not sure how to determine the coefficient. At first, she tried acting the Hamiltonian on the wave function. She rewrote the Hamiltonian in terms of the momentum and position operators and worked through the algorithmic procedures. Later, she realized that what she needed was a Fourier series.

Amy: So I think, you need a Fourier or sine series in order to get, I probably should have done a Fourier transform on my thing right away, because I could get my $\psi$ in terms of an infinite sum of things, infinite sum of energy eigenfunctions. I mean this was revealing, but I don't think that it actually helped me. I did bring to conclusion that I do need Fourier transform.

Amy realized that she was on the right track but did not recall how to determine the Fourier series. Although she used the term "Fourier transform," it is likely she meant Fourier series based on the rest of her interview.
The interviewer suggested moving on. After working through the entire task 1, the interviewer gave Amy several expressions, such as $|\psi\rangle, \psi(x)$, and $\langle\varphi \mid \psi\rangle$, one at a time. When asked to make sense of $\langle\varphi \mid \psi\rangle$, Amy said that she thought about inner products as dot products and interpreted dot products as the projections between spatial vectors.

Amy: Actually looking back, I think this is the idea of Fourier transform. All the sine(s) [energy eigenfunctions] are orthogonal, right? You do this dot product and everything cancels out except for the particular value you are looking for.

Sensemaking of the inner product $\langle\varphi \mid \psi\rangle$ appears to have produced an "aha" moment for Amy, who then realized how to determine the coefficient in task 1A. She realized that the inner product between the ground state and the given state vector is one of the coefficients in a Fourier series. She wrote down the inner product in Dirac notation and then immediately translated it into wave function notation as an integral. The high symbolic support of Dirac notation appears to have helped Amy make sense of inner products by forming an analogy to dot products of spatial vectors. Once she realized an inner product was needed, she was able to translate it from Dirac to wave function notation immediately.

Our results suggest that the high symbolic support of Dirac notation may help students determine the probability for an energy measurement when the wave function is not written as a linear combination of energy eigenfunctions. In particular, Dirac notation can be considered as providing strong visual cues to the interpretation of inner products. In Dirac notation, an unclosed bracket, |\rangle or \langle| , represents a vector in vector space, and a closed bracket, $\langle\mid\rangle$, represents a scalar. Thus, an expansion coefficient is picked out as a result of the closed bracket. The physical shape of the closed bracket, $\langle\mid\rangle$, can support students' ability to make a connection between the inner product and the probability by interpreting an inner product as the projection or overlap between two state vectors. Using Dirac notation as a template seems to be a productive strategy for determining energy probabilities.

\section{High compactness of Dirac notation may impede student ability to distinguish probability density from an inner product}

Although struggling for a while at the beginning, Nathan correctly answered both parts in task 1 . When asked whether the procedures he used to solve the two parts are similar or different, Nathan stated that they were different to him. He explained that in part A he took the "inner product of the state against the wave function" and in part B he took "an integral of probability density over the region of interest instead of the entire well." 
Int.: So you do not take inner product in part B like you did in part A?

Nathan: There's, I guess there's an inner product. I guess, this term [He points to $\left.\psi^{*} \psi\right]$ ], right there, is the inner product of the state with itself [He writes $\langle\psi \mid \psi\rangle]$. Yeah, Yeah! So it is an inner product I suppose. But in [part] A, inner product is sort of where it sucks out the value you're looking for cause it has energy eigenstate, the, the eigenvalue, yeah the eigenstate you are interested in, and then integrate over the whole well. Then the second one, you just inner product the state with itself and then look for specific region because you want the position, probability of position.

Int.: So what is the inner product of the state vector with itself? What is the result?

Nathan: Would be $\psi^{*} \psi$, which is real. Would be ... [He writes $\frac{30}{a^{5}}\left(x^{4}-2 a x^{3}+a^{2} x^{2}\right)$.]

Nathan did not seem to recognize that the wave function itself is the result of an inner product. Instead, he claimed that the probability density is an inner product. When asked for the result of the inner product of the state vector with itself, he plugged the wave function into the expression $\psi^{*} \psi$, which is actually the probability density.

An inner product in Dirac notation is highly compact. When the inner product $\langle\psi \mid \psi\rangle$ is translated into wave function notation, it is expressed as an integral $\int_{0}^{a} \psi^{*}(x)$ $\psi(x) d x$. Nathan did not seem to realize that the inner product (in Dirac notation) is equal to an integral (in wave function notation). The compactness of the inner product in Dirac notation conceals a highly relevant mathematical operation (the integral) of an inner product in wave function notation. The compactness of Dirac notation appears to impede Nathan's ability to relate the inner products in Dirac and wave function notation.

\section{Low externalization of Dirac notation may hinder student ability to interpret quantum states}

When Steve was determining the position probability on task $1 \mathrm{~B}$, he spontaneously wrote $\langle x \mid \psi\rangle=\int x \psi(x) d x$.

Int.: If we break down the inner product, the thing on the left, what would you call that?

Steve: The operator. It is the thing measuring $\psi$. I would technically put $\hat{x}$, but $\hat{x}$ is the same as $x$, which in that case, it's like what's the point of that? It makes more sense if we have $\hat{p} . \hat{p}$ is $-i \hbar d / d x$. This is not clearly, it's not like a regular mathematical function that you can just put in there. It has to be operating upon something, like your Hamiltonian kind of thing. Because we can have operator of position, momentum, Hamiltonian as our energy operator.

It seems that Steve associated the inner product with a measurement and with an operator. He incorrectly externalized the abstract symbol $\langle x|$ to something that he is (probably) familiar with: the position operator $\hat{x}$. Steve also compared it to a momentum operator to support his argument. Steve's interpretation of $\langle x|$ appears to be based only on the letter symbol that is used inside the bra rather than the entire bra notation. He related that symbol to a physical operation, a measurement, that is acted on the given state vector $|\psi\rangle$. Besides, he did not seem to differentiate between a measurement and an operator. It appears that the low externalization of Dirac notation hinders Steve's ability to make sense of inner product $\langle x \mid \psi\rangle$.

On the other hand, another student Tyson, seemed to interpret $\langle x|$ as a state during an interview. "So the way you would write it as an integral if you did, was like the state $x$ times $\psi d x$." Then he wrote $\langle x \mid \psi\rangle=\int x^{*} \psi(x) d x$, where $x^{*}$ represents the complex conjugate of $x$. It appears that Tyson recognized the bra $\langle x|$ as a state since he used the phrase "state $x$." However, he argued that this inner product could not be written as an integral.

Tyson: Yeah, there's no like state $x$.

Int.: There's no like state $x$ ?

Tyson: No, $x$ should be a position, right? It's not a state. Int.: It's not a state, then what does that, the half of the expression has a, it's a bra $x$, right? What does that mean?

Tyson: The bra part of it? That would indicate a complex conjugate or Hermitian conjugate.

Int.: Okay. What about a ket $x$ ? [The interviewer writes down $|x\rangle]$ What does that represent?

Tyson: Just $x$.

Int.: Just $x$. You mean this $x$ ? [The interviewer writes down X.] They are the same?

Tyson: Umm, yeah as far as I, that's what I was inclined to answer.

Int.: Okay. It's the same as function x, so you don't call this a state? That's not a state?

Tyson: No, it shouldn't be.

Tyson correctly recognized that an unclosed bracket should be a state and function $x$ does not represent a state (at least not a state that is commonly used in a junior-level textbook). However, he incorrectly believed that $|x\rangle$ is equal to function $x$ (which represents a position). Lastly, he concluded that this inner product could not be written as an integral because function $x$ does not describe a state.

It is perhaps not surprising that students attempt to externalize $|x\rangle$ to function $x$ since they share the same symbol. Although Steve and Tyson use different lines of reasoning, they both appear to externalize $\langle x|$ based on the symbol $x$ used in the bra. They both seem to confuse $x$ as a function and $x$ as a label either for the position operator or for an eigenstate of that operator. Again, the high compactness and low externalization of a state vector in Dirac 
notation may impede student ability to interpret quantum states during translation between notations.

We have evidence showing that students not only tend to externalize quantum states incorrectly, but also that they do not differentiate between a quantum state and a wave function denoted by the same letter symbol. Four out of five interviewees (one student was not asked this question) stated that they would call $|\psi\rangle$ a wave function [often expressed as $\psi(x)$ ]. Below is an example from Nathan's interview:

Int.: What does this expression $[|\psi\rangle]$ represent? Nathan: It $[|\psi\rangle]$ represents wave function of some kind of ket form.

Int.: Is it similar to or different from $\psi(x)$ ?

Nathan: I think they $[|\psi\rangle$ and $\psi(x)]$ are the same.

The wave function is denoted by a generic symbol $\psi$, which does not provide information about the specific form of the function. In this case, the expressions in both Dirac and wave function notations have a high degree of compactness but low degree of externalization. Nathan incorrectly believed that $|\psi\rangle$ is also a wave function just written in a different notation. It is very likely due to that both expressions are denoted by the same letter symbol $\psi$. This seems to be consistent with Tyson's belief that $|x\rangle$ is equal to $x$. This example lends support to understanding Tyson's belief. It appears that students tend to relate quantum state vectors to wave functions based on the symbol used in the kets (or the bras) and to believe those expressions are equivalent.

\section{DISCUSSION AND CONCLUSION}

This article describes an investigation into how students relate inner products and probabilities for discrete and continuous cases. The investigation was comprised of two stages with independent methods. We first examined student written work and reported the prevalence of correct and incorrect lines of reasoning. The results motivated our qualitative analysis of interview data through the lens of the structural features of quantum notations framework [25]. An overarching finding of this study is that many students do not seem to recognize that the method by which probabilities are determined is unified across all observables (regardless of whether the eigenvalues are discrete or continuous). In particular, both student written work and interview data suggest that (i) Dirac notation may support student sensemaking of inner products between energy eigenstates and the state vector, (ii) Dirac notation may hinder student interpretation of inner products between position eigenstates and the state vector, and (iii) unsuccessfully differentiating between a wave function and its associated state vector in Dirac notation may be a primary barrier for students to develop a model for determining probabilities for discrete and continuous cases. Below, we discuss how these findings are convergent from the student written work and interview data.

\section{A. Significance of differentiating between a wave function and its associated state vector}

Evidence from both exam and interview data suggest that using Dirac notation as a template supports student sensemaking of inner products between energy eigenstates and the state vector. Amy, who struggled to determine the coefficients of the wave function in the energy basis at the beginning, came to an aha moment when prompted to make sense of the inner product between two state vectors $\langle\varphi \mid \psi\rangle$. She was able to draw an analogy from the dot product of spatial vectors and then realized that the coefficients can be determined using inner products. This aha moment suggests that Dirac notation, due to its high symbolic support, can be helpful for students to determine (discrete) energy probabilities using inner products. The strong visual cue provided by the bracket $\langle\mid\rangle$ supports student sensemaking of inner products and their relations to probabilities. The results from the interviews are consistent with what we found from student written work: about three-quarters of the students who answered correctly spontaneously use Dirac notation for determining probabilities, even if the state of the particle is represented in wave function notation.

We found that although Dirac notation provides symbolic support for students to make sense of inner products between energy eigenstates and the state vector, the low externalization of Dirac notation appears to hinder student interpretation of position eigenstates $|x\rangle$. Tyson and Steve incorrectly externalized position eigenstates to the function $x$ and to the position operator $\hat{x}$, respectively. It appears that this externalization is based on the symbol used in the unclosed brackets. This error was also identified among students' written work: many students tend to match the symbols in the closed bracket to those in the integrals. In some textbooks, inner products are introduced using a generic expression: $\langle f \mid g\rangle=\int f^{*}(x) g(x) d x$. The error identified in this paper implies that using a generic expression without caution may lead students to generalize inappropriately.

Our results also suggest that the high compactness of Dirac notation can lead to some specific errors. Nathan incorrectly claimed that the probability density is the inner product between the state vector and itself. Inner products in Dirac notation are highly compact: an integral is encoded in the closed bracket. The high compactness of the inner products in Dirac notation seems to hinder student ability to differentiate between $\langle\psi \mid \psi\rangle$ and $\psi^{*}(x) \psi(x)$. This seems consistent with common errors we have identified among students' written work: many students (i) believe that $\langle\psi \mid \psi\rangle$ is equal to $\psi^{*}(x) \psi(x)$, and (ii) appear to think $\varphi_{2}(x) \varphi_{3}(x)$ equals zero due to the orthorgonality of energy eigenstates.

The errors discussed above point to an underlying barrier for students to develop a coherent model for determining 
probabilities in discrete and continuous cases: not differentiating between a state vector and its corresponding wave function. In addition, we have found that many students expressed equivalence between state vectors and wave functions during individual interviews and written tasks. Those students stated that they would call both $|\psi\rangle$ and $\psi(x)$ a wave function. This specific error was documented in Gire and Price [25]. They argued that this error may be a productive intermediate step in using Dirac notation as a template. We agree that when students compute the expectation value for energy, closely associating a state vector and its associated wave function may be a productive intermediate step. However, when students reason about quantum states and inner products, this error appears to lead to other common errors. In order to recognize the methods for determining (discrete) energy and position probabilities are analogous, it is a key step to understand that wave function $\psi(x)$ is equal to the inner product $\langle x \mid \psi\rangle$ rather than the state vector $|\psi\rangle$. Therefore, we argue that failing to differentiate between a state vector and its corresponding wave function may be a primary barrier for developing a model for determining probabilities.

\section{B. Comparing findings of structural features on student computations and sensemaking}

This paper demonstrates how the structural features of quantum notations framework [25] can be applied to analyze how students make sense of quantum mechanics. The results suggest that several structural features of Dirac and wave function notations appear to have an impact on student sensemaking about quantum states and inner products. We compare our findings to what have been documented in Gire and Price [25], who developed the theoretical framework to analyze student performing computations.

The results discussed in this paper suggest that the high individuation of wave function notation in the position basis may support students in determining position probabilities. However, the low symbolic support of wave function notation appears to hinder student ability to determine energy probabilities. In contrast, Dirac notation, due to its high degree of symbolic support, may help students make sense of inner products between the energy eigenstates and the state vector. This is consistent with Gire and Price, who found that using Dirac notation as a template is a productive strategy for students to compute the expectation value for energy [25]. It appears that Dirac notation not only facilitates computation, but also supports sensemaking of some abstract expressions.

Our results also show that the high compactness and the low externalization of Dirac notation, in some other cases, appear to hinder student sensemaking of quantum states and inner products such as $\langle\psi \mid \psi\rangle$ and $\langle x \mid \psi\rangle$. Gire and Price, on the other hand, found that students easily generate expressions for expectation value for energy in Dirac notation due to its high compactness. In addition, they argued that the low externalization of Dirac notation can be helpful when students do not remember that the energy eigenfunctions for the infinite square well are sine functions. Our results and the findings from Gire and Price together imply that certain structural features that are considered as supporting computation can hinder sensemaking. It is possible that the structures of the expressions (an expectation value like $\langle\psi|\hat{H}| \psi\rangle$ versus an inner product like $\langle\psi \mid \psi\rangle)$ may also have an impact on student performance. However, the extent to which certain features of the different notations supports or hinders student reasoning also seems to depend on the specific nature of the task (i.e., whether it requires computation or sensemaking).

\section{Limitations}

One important limitation of this study may be that the tasks only involve certain contexts and prompts, thus the results may not apply to other tasks. The written tasks used only involve energy and position probabilities. It is possible that some of the common errors and lines of reasoning identified do not apply to other contexts, such as spin and angular momentum. The interview tasks were designed based on the written tasks but focus more on student sensemaking. Only certain but not all structural features were identified as supporting or hindering student reasoning. For example, the data does not show how the externalization of wave function notation can impact student reasoning. Therefore, this paper does not provide a comprehensive analysis of each of the structural features.

Another important limitation may be the representativeness of the participants. For two of the three written tasks, only about half of the students enrolled participated in this study. The participants may not form a well-representative sample. Although the data from different academic quarters are not statistically significantly different, it could be that the participants represent a particular group of students. Similarly, the interview participants are subject to a selfselection effect. All the interviewees came from the top half of the class in terms of their final course grades, although we did not select them on this basis.

\section{Instructional implications}

The findings discussed in this article have been informing our efforts to improve students' ability to determine quantum probabilities. We have been developing tutorial curriculum [30] to help students construct a coherent model for quantum states and inner products across the different common notations. During the process of curriculum development, we have been accounting for the affordances and limitations of the notations due to their structural features. To support student conceptual understanding and mastery of formalism, the tutorials (i) use Dirac notation to facilitate student sensemaking about inner products (e.g., to interpret $\langle x \mid \psi\rangle$ as a scalar projection, which is analogous 
to a dot product between vectors in laboratory space); (ii) help students interpret $\psi(x)$ as an expansion coefficient in the position basis, which is analogous to an expansion coefficient in the energy basis; and (iii) guide students to relate inner products in Dirac and wave function notations and to make sense of the expressions in these notations. Furthermore, the tutorials not only carefully disambiguate the terms "wave function" and "state vector," but also actively question students about the differences between them. Future research will evaluate the effectiveness of these tutorial curricula. The instructional strategy used in the tutorial curricula puts an emphasis on making sense of the mathematical formalism of quantum mechanics.
We propose that including this sensemaking across all quantum instruction, including lecture instruction, will help facilitate students' functional understanding of the formalism.

\section{ACKNOWLEDGMENTS}

The authors would like to acknowledge the valuable input from Lisa Goodhew, Jason Detwiler, and Elizabeth Gire on this manuscript. We thank the UW faculty members who welcomed this research in their courses. Last but not least, we thank all the students who participated in this study, especially those who volunteered for the interviews.
[1] D. J. Griffiths, Introduction to Quantum Mechanics, (Pearson Prentice Hall, Upper Saddle River, NJ, 2005).

[2] J.S. Townsend, A Modern Approach to Quantum Mechanics, University Science Books, 2nd ed. (2012).

[3] D. H. McIntyre, Quantum Mechanics: A Paradigm Approach, 1st ed. (Addison-Wesley, Boston, MA, 2012).

[4] C. Singh, Student understanding of quantum mechanics, Am. J. Phys. 69, 885 (2001).

[5] E. Marshman and C. Singh, Framework for understanding student difficulties in quantum mechanics, Phys. Rev. ST Phys. Educ. Res. 11, 020119 (2015).

[6] C. Singh and E. Marshman, Review of student difficulties in quantum mechanics, Phys. Rev. ST Phys. Educ. Res. 11, 020117 (2015).

[7] P. J. Emigh, G. Passante, and P. S. Shaffer, Student understanding of time dependence in quantum mechanics, Phys. Rev. ST Phys. Educ. Res. 11, 020112 (2015).

[8] G. Passante, P. J. Emigh, and P. S. Shaffer, Examining student ideas about energy measurements on quantum states across undergraduate and graduate levels, Phys. Rev. ST Phys. Educ. Res. 11, 020111 (2015).

[9] P. J. Emigh, G. Passante, and P. S. Shaffer, Developing and assessing tutorials for quantum mechanics: Time dependence and measurements, Phys. Rev. Phys. Educ. Res. 14, 020128 (2018).

[10] H. Close, C. Schiber, E. Close, and D. Donnelly, Students dynamic geometric reasoning about quantum spin-1/2 states, in Proceedings of the 2013 Physics Education Research Conference, Portland, OR (AIP, New York, 2013), pp. 93-96.

[11] T. Wan, P. J. Emigh, and P. S. Shaffer, Student understanding of the measurable effects of relative phases in superposition states, in Proceedings of the 2017 Physics Education Research Conference, Cincinnati, OH (AIP, New York, 2017), pp. 420-423.

[12] E. Gire and C. A. Manogue, Making sense of quantum operators, eigenstates and quantum measurements, AIP Conf. Proc. 1413, 195 (2012).

[13] A. Crouse, Ph.D. thesis, University of Washington, Seattle, Washington, 2007.
[14] G. Zhu and C. Singh, Surveying students understanding of quantum mechanics in one spatial dimension, Am. J. Phys. 80, 252 (2012).

[15] G. Zhu and C. Singh, Improving students understanding of quantum measurement I: Investigation of difficulties, Phys. Rev. ST Phys. Educ. Res. 8, 010117 (2012).

[16] G. Zhu and C. Singh, Improving students understanding of quantum measurement II: Development of research-based learning tools, Phys. Rev. ST Phys. Educ. Res. 8, 010118 (2012).

[17] H. Sadaghiani and L. Bao, Student difficulties in understanding probability in quantum mechanics, AIP Conf. Proc. 818, 61 (2006).

[18] C. Singh, Student understanding of quantum mechanics at the beginning of graduate instruction, Am. J. Phys. 76, 277 (2008).

[19] P. J. Emigh, Ph.D. thesis, University of Washington, Seattle, Washington, 2015.

[20] C. Singh, Student difficulties with quantum mechanics formalism, AIP Conf. Proc. 883, 185 (2007).

[21] T. Wan, Ph.D. thesis, University of Washington, Seattle, Washington, 2018.

[22] C. Singh and E. Marshman, Investigating student difficulties with Dirac notation, in Proceedings of the 2013 Physics Education Research Conference, Portland, OR (AIP, New York, 2013).

[23] E. Marshman and C. Singh, Student difficulties with quantum states while translating state vectors in Dirac notation to wave functions in position and momentum representations, in Proceedings of the 2015 Physics Education Research Conference, College Park, MD (AIP, New York, 2015).

[24] E. Marshman and C. Singh, Investigating and improving student understanding of the probability distributions for measuring physical observables in quantum mechanics, Eur. J. Phys. 38, 025705 (2017).

[25] E. Gire and E. Price, Structural features of algebraic quantum notations, Phys. Rev. ST Phys. Educ. Res. 11, 020109 (2015). 
[26] P. A. M. Dirac, A new notation for quantum mechanics, Math. Proc. Cambridge Philos. Soc. 35, 416 (1939).

[27] S. Goldhaber, S. J. Pollock, M. Dubson, P. Beale, and K. K. Perkins, Transforming upper-division quantum mechanics: Learning goals and assessment, AIP Conf. Proc. 1179, 145 (2009).

[28] https://physicscourses.colorado.edu/EducationIssues/ Quantum/learning_goals.html. Retrieved 1/15/2019.

[29] A. F. Heckler and T. M. Scaife, Adding and subtracting vectors: The problem with the arrow representation, Phys. Rev. ST Phys. Educ. Res. 11, 010101 (2015).
[30] L. C. McDermott, P. S. Shaffer, P. R. L. Heron, and the Physics Education Group, Tutorials in physics: Quantum mechanics (to be published).

[31] Student consent to participating to the study was not required in this academic year.

[32] This student wrote the expression in Dirac notation correctly and had the correct integrand in wave function notation. However, the limits of the integral were not correct. This response was categorized as a correct answer considering there was only a minor mistake. 